\title{
Transformaciones en el pensamiento de unos profesores universitarios de áreas no lingüísticas sobre la escritura académica
}

\author{
Isabel Martins \\ Universidad Simón Bolívar, Caracas, Venezuela
}

Artículo recibido 19 de enero de 2012; aceptado 15 de febrero de 2013; version final recibida 28 de marzo de 2014

\begin{abstract}
Este artículo avanza algunos resultados sobre las transformaciones en los sistemas de creencias, representaciones y saberes (Cambra, 2000) respecto a la escritura académica y su pedagogía de tres profesores de diversas áreas en un taller de formación en alfabetización académica en la Universidad Simón Bolívar (Caracas, Venezuela). Esta intervención formativa adaptó algunos principios de la tercera generación de la teoría de la actividad y el aprendizaje expansivo (Engeström, 1987; Engeström y Sannino, 2010) y de la teoría de los sistemas de géneros (Bazerman, 1994). Como investigadorainterventora y sujeto del mismo sistema de actividad que los informantes, interpreto sus concepciones a partir de los discursos escritos generados antes y después de sesiones de trabajo planificadas ad hoc. El análisis de los datos es interpretativo como el que ofrece la teoría de la enunciación (Palou, 2008). Palabras clave: formación profesoral, pensamiento del profesor, alfabetización académica, teoría de la actividad, teoría de los géneros discursivos.
\end{abstract}

\section{Introducción}

El ámbito temático de esta investigación es la alfabetización académica ${ }^{\mathrm{i}}$. Ahora bien, es importante aclarar que en el contexto venezolano no podemos entender que se trata de un desarrollo similar al de los movimientos internacionales de formación lingüística universitaria intra y extracurriculares del mundo anglosajón (véase Russell, Lea, Parker, Street y Donahue, 2009). De hecho, si revisamos las decisiones políticas que llevaron a crear este tipo de movimientos, así como las líneas maestras que los sustentan y sus progresos en la práctica, podemos asegurar que tal tipo de experiencia es prácticamente inexistente en Venezuela.

Serrón (2004) señala que desde la pasada década de los 70 se han realizado importantes esfuerzos educativos e investigativos sobre la escritura académica en Venezuela, sin embargo asegura que sus efectos han sido insuficientes. En otra publicación, este autor (2007, p. 3) insiste en que la preocupación por encontrar una fórmula que haga del estudiante universitario y del graduado, un usuario competente de su lengua materna "está lejos de dar frutos"; por lo que hace un llamado a las administraciones públicas y universitarias a comprometerse con nuevas propuestas educativas. Por su parte, Serrano (2011) también 
discute sobre la responsabilidad institucional respecto a la escritura académica en la Universidad de Los Andes, una de las más importantes casas de estudio del país. Esta autora indica que a pesar de que en su universidad existen desde hace más de veinte años programas de postgrado en el área de la lengua escrita, la preocupación por estudiar la escritura académica universitaria es reciente y ha sido atendida solamente por unas cuantas iniciativas individuales.

En definitiva, es evidente la configuración de un panorama problemático. En este sentido, este trabajo se justifica como un marco de conocimiento necesario para proponer, discutir e implementar políticas institucionales de formación lingüística tanto para profesores como para estudiantes en la Universidad Simón Bolívar (USB, en adelante). El aporte fundamental de nuestro trabajo es hacer visibles los pensamientos de tres profesores de distintas áreas profesionales (no lingüísticas) para incorporar sus "voces” (Bajtín, 1989) como materia prima al debate sobre la enseñanza de la escritura en lengua materna en las universidades venezolanas. Se trata de un estudio de caso para hacer explícitos los cambios en los significados que poseen los sujetos participantes de la actividad formativa cuya finalidad fue redescribir sus representaciones (Pozo, 1999) sobre la enseñanza de la lengua escrita.

Los objetivos de este trabajo están estrechamente vinculados con el concepto de Vigotsky (1977) de la Zona de Desarrollo Próximo (ZPD, en adelante). Engeström (1987) redefine este concepto para tratar el aprendizaje en el nivel de las actividades colectivas. Desde su perspectiva, la ZDP corresponde a la distancia entre las acciones de los individuos en el presente y una forma históricamente nueva de la actividad social que puede generarse de modo colectivo como una solución al dilema potencialmente inherente a las acciones de cada día (Engeström y Sannino, 2010). Tomando en cuenta estas premisas, el objetivo general del estudio es describir en profundidad cómo se transforman los sistemas de creencias, representaciones y saberes (CRS, en adelante) (Cambra, 2000) de un grupo de tres profesores de distintas áreas profesionales no lingüísticas sobre la escritura académica y su pedagogía en el contexto de una intervención formativa en la USB.

En cuanto a los objetivos específicos, nos planteamos los siguientes: 1) Identificar y caracterizar en el contexto del taller “¿Cómo incorporar al aula la escritura como herramienta de aprendizaje de las disciplinas universitarias y profesionales?", mediado por la investigadora-interventora, la ZDP de un colectivo de profesores de distintas áreas 
profesionales sobre qué es la lengua y cómo debe ser enseñada y aprendida en la universidad. 2) Identificar y caracterizar en profundidad cuáles son los elementos que promueven el cambio en los sistemas de CRS (Cambra, 2000) de un grupo de profesores de distintas áreas profesionales sobre la enseñanza de la lengua en la universidad, con base en el diálogo reflexivo y en la incorporación de nuevas experiencias de formación en alfabetización académica.

\section{Marco de referencia}

En este estudio, adapté e integré la teoría de la actividad y el aprendizaje expansivo, y los estudios sobre el pensamiento del profesor. La primera propone una unidad de análisis sobre la actividad humana, un proceso dinámico en donde unos sujetos comparten un mismo objeto/motivo para lograr un resultado, mediante diversas herramientas materiales y simbólicas en una comunidad determinada por la división del trabajo y reglas. A este enfoque global de la actividad, integré la segunda teoría para aproximarnos al componente cognitivo de los sujetos mediante el análisis de la interacción comunicativa.

Para Engeström (1996), el modelo de la actividad humana fue un gran paso hacia adelante porque cambió el foco de las complejas interrelaciones entre el sujeto individual y su comunidad. En esta misma publicación, Engeström detalla tres principios de tipo metodológico para analizar e interpretar los datos que registran y describen el comportamiento y los discursos humanos: 1) un sistema de actividad colectiva puede tomarse como unidad de análisis para aportar contexto y significado a las acciones individuales; 2) el sistema de actividad y sus componentes pueden ser entendidos desde el punto de vista histórico; y 3) las contradicciones internas del sistema de actividad (incluidos sus participantes individuales) pueden comprenderse como fuente de desorganización, innovación, cambio y desarrollo. Asimismo, resalta que entre los componentes de un sistema de actividad, la transformación prosigue sin cesar; de esta manera, los seres humanos no sólo usan los instrumentos sino que (a sabiendas o no) los renuevan y desarrollan constantemente. Es decir, no sólo obedecen reglas, también las moldean y reformulan. Esta dialéctica ofrece la posibilidad del aprendizaje expansivo. Engeström y Sannino (2010) apuntan que este tipo de aprendizaje permite a los aprendices involucrarse en la construcción e implementación de un objeto nuevo más amplio y más complejo, y de un concepto para su actividad; de este modo, ellos aprenden algo que todavía no existe. 
Desde la perspectiva de la Nueva Retórica, Bazerman (1994) ubica leer y escribir como las acciones tipificadas construidas en sistemas de la actividad complejos. Según este autor, en la medida en que conocemos y nos involucrados con este conjunto de géneros discursivos, podemos compartir de manera más precisa sus significados y sus relaciones funcionales. Desde su punto de vista, mediante el uso de estos textos tipificados somos capaces de hacer avanzar nuestros propios intereses y dar forma a nuestros significados en relación con los sistemas sociales complejos, así como somos capaces conferir valor y resultado a las declaraciones de los demás. Para ilustrar su definición este autor apunta que los sistemas de géneros son como una "máquina" de la sociedad en la que los géneros discursivos forman "palancas" importantes. Tales "palancas" debemos poder reconocerlas, usarlas y construirlas cercanas a las de su tipo con el fin de crear la acción social consecuente. Y agrega que esta "máquina" sólo se queda trabajando en la medida en que participamos en ella y hacemos nuestras vidas a través de sus géneros, precisamente porque los géneros nos permiten crear significados en los sistemas articulados y altamente desarrollados de la sociedad.

Sobre el pensamiento del profesor, Cambra (2000) y el grupo de investigación PLURAL de la Universidad de Barcelona se han dedicado a explorar las creencias de los profesores de lengua(s) porque estas tienen un efecto sobre cómo perciben su actividad y sobre los comportamientos en la clase. Su principal aporte teórico es el constructo CRS, cuyas características principales son: está determinado y se desarrolla por contextos de experiencia; es personal, particular y específico; a veces es compartido por un grupo social determinado; constituye un marco para controlar la complejidad presente en el aula; se mantiene implícito e incluye elementos conscientes y otros que no lo son; es una síntesis de elementos provenientes de distintos campos del saber; está poco organizado y es construido con elementos anecdóticos; es un marco de referencia tácito para la acción; aunque es resistente al cambio, puede evolucionar a causa de nuevas experiencias; y corresponde frecuentemente a clichés estereotipados (Cambra, 2000).

En el ámbito de las investigaciones publicadas en el contexto anglosajón, Borg (2003, p. 81) usa el término "teacher cognition" para referirse a la dimensión cognitiva no observable de la enseñanza, es decir, lo que los profesores saben, creen y piensan. Este autor realiza un mapa que abarca los últimos veinticinco años de las investigaciones sobre esta área del conocimiento. Estos estudios han confirmado que los profesores son activos al pensar y 
tomar decisiones sobre las opciones de instrucción mediante el uso de complejas redes conocimientos, pensamientos y creencias, orientadas a la práctica, personalizadas y sensibles al contexto. Sobre la terminología empleada en el campo de la cognición docente, Borg (2003) se refiere a que existen similitudes importantes en el contenido de las etiquetas usadas por los investigadores del área; en particular, las siguientes: la naturaleza personal de la cognición docente; el papel de la experiencia en su desarrollo; y la forma en que la práctica docente y la cognición se informan mutuamente.

\section{Opción metodológica del estudio}

La orientación cualitativa fue la opción metodológica elegida para este estudio. Esta se interesa por cómo las personas viven, experimentan, interpretan y construyen los significados del mundo social, y cómo estos significados se integran en la cultura, el lenguaje y las acciones de los actores sociales (Latorre, del Rincón y Arnal, 1996). A la par, existe la intención etnográfica. Además, consideramos uno de los planteamientos centrales de Bajtín (1989) sobre la enunciación y los géneros discursivos en relación con el lenguaje; en sus propias palabras: "El lenguaje participa de la vida a través de los enunciados concretos que lo realizan, así como la vida participa del lenguaje a través de los enunciados” (p. 251). En nuestro caso, se trata de describir desde la perspectiva de unos informantes clave la tradición que ellos han heredado, y cómo esta se transforma, que se manifiesta a través de sus discursos.

\section{Contexto}

La realización del taller en alfabetización académica en la USB fue el contexto que permitió favorecer el intercambio (diálogo reflexivo) sobre los temas de interés entre diferentes subsistemas de actividad académicos: los de los profesores participantes y el de la investigadora. En términos de la teoría de la actividad y el aprendizaje expansivo, se buscó propiciar el "cruce de fronteras" (Engeström y Sannino, 2010, p. 12) a través de un espacio ya existente en la institución: los programas educativos de la Dirección de Desarrollo Profesoral, cuyo propósito es brindar formación especializada al personal académico. Dicho taller (véase el cuadro 1) se dictó durante un trimestre en el año 2011. Su diseño y aplicación adaptó los principios y herramientas metodológicas del aprendizaje expansivo: el llamado "laboratorio de cambio" a modo de "micro ciclo de innovación" para provocar las 


\section{interacciones entre los participantes, que fueron mediadas por la investigadora-interventora con miras a lograr el aprendizaje expansivo (Engeström y Sannino, 2010).}

Cuadro 1: Aspectos relevantes del programa del taller “¿Cómo incorporar al aula la escritura como herramienta de aprendizaje de las disciplinas universitarias y profesionales?"

\section{Presentación}

Desde hace décadas se llevan a cabo alrededor del mundo (particularmente en países de habla inglesa, como Australia, Estados Unidos, Canadá e Inglaterra) cursos de enseñanza de la lectura y escritura en las diversas disciplinas universitarias, especializadas (científicas) y profesionales. Dos principios básicos sustentan este tipo de actividad de formación. Primero, al ser la universidad un ámbito de actividades discursivas especializadas es necesario hacer explícitos a los estudiantes los conocimientos (teóricos y prácticos) sobre esta nueva cultura; y segundo, se valora de modo especial la escritura como una herramienta potente de aprendizaje. El curso que propongo servirá a los profesores participantes para familiarizarse con los aspectos teóricos básicos de la alfabetización académica y los ejemplos puestos en práctica en el mundo, de manera tal que puedan ser adaptados al contexto uesebista para ser aplicados a través de propuestas didácticas específicas según el área de conocimiento de cada docente.

\section{Participantes}

Profesores (as) tanto del ciclo básico como del profesional y de cualquier disciplina que deseen potenciar el aprendizaje de sus estudiantes mediante la incorporación de la lectura y escritura a las actividades de aula y de evaluación.

\section{Finalidad}

A partir de la experiencia y conocimiento de los profesores participantes sobre la o las materias que imparten, así como de las generadas durante este curso, redescribir sus concepciones sobre la enseñanza de la lectura y la escritura en la universidad y en las disciplinas (alfabetización académica).

\section{Objetivos}

1. Ofrecer información sobre los fundamentos y programas de alfabetización académica en el mundo.

2. Valorar las posibilidades que ofrecen las actividades de lectura y escritura en las distintas disciplinas.

3. Asesorar a los participantes para que realicen una propuesta didáctica de alfabetización académica, una programación de actividades de lectura y escritura en sus respectivos cursos relacionadas con sus particulares ámbitos de conocimiento.

\section{Horario}

Su duración es de 24 horas académicas (12 sesiones de dos horas), 12 horas serán presenciales (en las semanas pares) y las otras 12 horas a distancia (en las semanas impares). La modalidad presencial servirá para discutir la teoría o evaluar la práctica; mientras que la modalidad a distancia será para realizar las tareas individuales (o de grupo) como la lectura de textos y las actividades relacionadas con la propuesta de intervención didáctica con el apoyo de la instructora, empleando para ello las tecnologías de comunicación (correo electrónico, foro, chat, blog, etc.)

\section{Temario}

1. Alfabetización académica: la concepción tradicional de la enseñanza de la lengua en la universidad; lectura y escritura como herramientas de aprendizaje e incorporación a las culturas académica, disciplinares y profesionales; ejemplos de programas exitosos en el mundo anglosajón e en el hispano.

2. Alfabetización académica en la USB: formación lingüística previa de los estudiantes y en los ciclos básico y profesional; necesidades educativas académicas y necesidades profesionales de los estudiantes; posibilidades de actuación.

3. Propuestas de intervención didáctica en la USB con base en actividades de lectura y escritura vinculadas con el ámbito temático y las competencias lingüísticas exigidas en cada disciplina.

\section{Metodología}

Estas etapas cubren tanto las horas presenciales como las a distancia: 
1. Procesamiento de la información de las lecturas obligatorias y contactos previos con la instructora.

2. Discusión a partir de las lecturas correspondientes a los fundamentos conceptuales sobre la alfabetización académica.

3. Discusión de casos particulares de intervenciones didácticas exitosas de alfabetización académica en el mundo, así como sus posibilidades reales de aplicación en el ámbito uesebista.

4. Recogida y procesamiento de datos relevantes (portafolio digital) de cada participante (o grupos de ellos) sobre el discurso escrito de su disciplina y sus modos de enseñarlo.

5. Elaboración de una propuesta de intervención didáctica de alfabetización académica adaptada al curso que dicte cada participante (o grupo de ellos).

6. Retroalimentación a los participantes por parte de sus compañeros y de la instructora sobre cada una las propuestas de intervención didáctica de alfabetización académica en la USB.

\section{Recolección de datos}

Cada participante redactó dos textos reflexivos, uno al inicio y otro al final del taller. Según Palou (2011, pp. 65-66), lo que se intenta con ellos es que "el pensamiento se refleje a sí mismo, a través del lenguaje", ya que "ofrecen un tiempo y un espacio para que el sujeto se haga cargo de los cambios que configuran su propia historia cognitiva." Al analizar el tipo de intercambio comunicativo que subyace en los textos reflexivos solicitados, tenemos que se presentan dos interlocutores de acuerdo al rol que cumplen en la actividad formativa: la investigadora-interventora y los participantes. Es la primera quien dirige a los segundos, en términos de que fija ciertos límites de su actuación verbal. En concreto, las indicaciones ofrecidas para el escrito inicial fueron: Escribe a continuación un texto reflexivo que refleje tu percepción sobre la actividad de escribir en la universidad. La idea es que expreses con absoluta libertad tus ideas previas sobre este asunto. Estas ideas pueden ser de diversa naturaleza: informaciones, opiniones, experiencias, dudas, etc. Es decir, intenta que tu texto recoja lo que conoces, intuyes, sientes, supones y/o te inquieta sobre el tema. Pueden abarcar tu propia tarea de escritor, la de tus colegas y/o la de tus estudiantes. También, la extensión y tipo de texto (formato o género) lo dejo a tu criterio. Respecto al texto reflexivo final, fue solicitado en el aula al inicio de la última sesión presencial del taller, y en estos términos: Con la experiencia del taller, redacta un texto sobre cómo percibes ahora la actividad de escribir en la universidad (o alfabetización académica). La idea es que expreses de un modo personal y con absoluta libertad tu parecer sobre este asunto a partir de tus experiencias y de las reflexiones suscitadas en esta intervención didáctica. La extensión y tipo de texto (formato o género) lo dejo a tu criterio.

\section{Análisis de los datos}


Se aplicó el modelo de análisis del discurso que utiliza el grupo de investigación PLURAL, basado en los estudios sobre las características del discurso en interacción de KerbratOrecchioni (2005, citada por Palou, 2008). En el cuadro 2, sintetizo el proceso de análisis e interpretación llevado a cabo que corresponde a una adaptación de la propuesta de Palou (2010; véase también Palou y Fons, 2009).

Cuadro 2: Proceso de análisis e interpretación de los textos reflexivos escritos

\begin{tabular}{|c|c|c|}
\hline PROCESO & INSTRUMENTOS & \multirow[b]{2}{*}{ ACCIONES Y HERRAMIENTAS SUBSIDIARIAS } \\
\hline \multirow{4}{*}{$\begin{array}{l}\text { PRIMERA } \\
\text { APROXIMACIÓN } \\
\text { Análisis discursivo }\end{array}$} & $\begin{array}{l}\text { Dimensiones } \\
\text { y sus indicadores }\end{array}$ & \\
\hline & $\begin{array}{l}\text { Interlocutiva: } \\
\text { Género discursivo } \\
\text { Conducta discursiva }\end{array}$ & $\begin{array}{l}\text { Describí el texto reflexivo como un género discursivo, } \\
\text { mediante el análisis de las particularidades estructurales, } \\
\text { formulación característica, aspectos de contenido temático, } \\
\text { condiciones situacionales y función comunicativa. } \\
\text { Describí la conducta discursiva a partir de las características } \\
\text { de los modos de organizar el discurso: narrar, describir, } \\
\text { explicar y argumentar. }\end{array}$ \\
\hline & $\begin{array}{l}\text { Temática: } \\
\text { Resumen } \\
\text { Palabras claves } \\
\text { Constelación de } \\
\text { palabras }\end{array}$ & $\begin{array}{l}\text { Ubiqué las palabras clave y determiné el conjunto de las que } \\
\text { fueran semánticamente próximas. } \\
\text { Determiné la macroestructura semántica, lo que incluye el } \\
\text { tema global y los temas locales. Por último, escribí una } \\
\text { versión reducida. }\end{array}$ \\
\hline & $\begin{array}{l}\text { Enunciativa: } \\
\text { Modalidad de la } \\
\text { oración } \\
\text { Modos del verbo y } \\
\text { perífrasis verbales } \\
\text { Locuciones, frases } \\
\text { hechas } \\
\text { Metáforas } \\
\text { Pronombres } \\
\text { Elementos léxicos } \\
\text { valorativos } \\
\text { Ahora y antes } \\
\text { Puntos críticos } \\
\end{array}$ & $\begin{array}{l}\text { Interpreté los significados de las diversas categorías } \\
\text { gramaticales y también de los recursos supraoracionales para } \\
\text { identificar la posición de quien escribe sobre lo que dice. }\end{array}$ \\
\hline $\begin{array}{l}\text { SEGUNDA } \\
\text { APROXIMACIÓN } \\
\text { Síntesis } \\
\text { interpretativa }\end{array}$ & $\begin{array}{l}\text { Análisis } \\
\text { interpretativo }\end{array}$ & $\begin{array}{l}\text { En función de los objetivos específicos de la investigación, } \\
\text { integré en un comentario interpretativo los diferentes } \\
\text { aspectos analizados. Respecto al texto reflexivo final, esta } \\
\text { síntesis incluye el contraste con el escrito inicial de cada } \\
\text { participante. }\end{array}$ \\
\hline
\end{tabular}

\section{Avance de resultados}

En este apartado presento los resúmenes de los dos textos reflexivos de cada participante, a quienes hemos nombrado como: "Homero", "Margarita" y "Pedro", para cumplir con la cláusula de confidencialidad, así como también, sendos comentarios interpretativos a partir del contraste de estos textos atendiendo a las transformaciones en su pensamiento sobre la escritura académica y su pedagogía en el contexto de la intervención formativa. 


\section{Homero}

Cuadro 3: resúmenes de los textos reflexivos escritos por Homero

\begin{tabular}{|c|c|}
\hline Inicial & Final \\
\hline $\begin{array}{l}\text { Gira en torno a la definición y valoración de dos objetos: } \\
\text { la escritura como una herramienta fundamental de la } \\
\text { sociedad y del pensamiento; y la evaluación de la } \\
\text { escritura de sus estudiantes. Sobre esto último detalla los } \\
\text { problemas: ciertas imprecisiones, errores ortográficos y } \\
\text { de sintaxis; y sus causas: su condición de "nativos } \\
\text { digitales" y la educación básica recibida. Por último, } \\
\text { ofrece sus propuestas: escribir sin computadoras, exigir } \\
\text { más y proporcionar material documental. }\end{array}$ & $\begin{array}{l}\text { Comienza con una valoración positiva de su } \\
\text { experiencia en el taller; luego, como resultado de un } \\
\text { proceso reflexivo, reconoce sus propias dificultades } \\
\text { en la escritura desde sus inicios como profesor. } \\
\text { Asimismo, admite que ha aprendido de sus } \\
\text { estudiantes, aunque ellos también evidencian fallas } \\
\text { en la escritura. Por último, señala el efecto negativo } \\
\text { de la cultura digital y evalúa su trabajo como } \\
\text { docente por contrarrestar tal efecto. }\end{array}$ \\
\hline
\end{tabular}

En su texto final, observamos la incorporación de reflexiones sobre sus propias dificultades como escritor. También emplea una metáfora para referirse a sus alumnos: ellos han sido "sus mejores maestros". Sin embargo, ninguno de estos cambios está relacionado con las herramientas teóricas (y sus posibilidades de aplicación) revisadas y discutidas en las sesiones de la actividad formativa. En este sentido, la evolución de sus concepciones sobre la escritura académica y su pedagogía en términos de su actividad educativa es poco visible, ya que en su escrito final nuevamente hace referencia a las debilidades de sus estudiantes como aspecto problemático y manifiesta las mismas soluciones. Asimismo, es reiterativa la mención que hace sobre la cultura digital como la causa que explicaría la baja calidad de los escritos que evalúa.

\section{Margarita}

Cuadro 4: resúmenes de los textos reflexivos escritos por Margarita

\begin{tabular}{|l|l|}
\hline \multicolumn{1}{|c|}{ Inicial } & \multicolumn{1}{|c|}{ Final } \\
\hline Se centra en distintos momentos de su vida & Hace un reporte de los aprendizajes obtenidos en el \\
lingǘstica: el pasado (adolescencia y vida estudiantil & taller. Estos aprendizajes se centran en la palabra clave: \\
universitaria); presente (su rol como asesora y & reflexión. A partir de esta idea, detalla cuáles han sido \\
docente); y futuro próximo (proyectos de & las acciones puestas en práctica en su labor docente; \\
publicaciones en su área profesional). Además, & señala las principales dificultades de sus estudiantes y \\
valora la escritura como aspecto fundamental para un & también algunas reservas sobre su trabajo con ellos. \\
desempeño profesional exitoso. & \\
\hline
\end{tabular}

Su escrito inicial consistió en una biografía lingüística sobre cómo fue su relación con la lengua escrita a lo largo de las distintas etapas de su vida. En contraste, su texto final corresponde a la evaluación de los aprendizajes obtenidos en el taller, la descripción de las acciones emprendidas y las dificultades que se le han presentado en su práctica docente. Llama la atención que al comienzo del taller se muestre (aunque intuitivamente) más cercana a los principios de la alfabetización académica que al final, ya que su experiencia como 
escritora a lo largo de su vida la llevó a adaptarse a los diversos contextos específicos de actuación lingüística, lo que le hizo desarrollar una competencia genérica para adaptarse a cada entorno de uso lingüístico: académico (como estudiante) y profesional (como ingeniera y como docente). Sin embargo, en su texto final este conocimiento, clave en las discusiones a lo largo del taller, no aparece reflejado en su actuación en el aula. Lo implementado corresponde a su propia actuación como escritora, o a operaciones como la incorporación de nuevos términos al vocabulario de los estudiantes y la consulta de diversas fuentes para complementar lo dicho en clase. Se trata de la puesta en práctica de actividades que ella denomina "del colegio" y no corresponden a una intervención didáctica más integral y de mayor alcance como las abordadas en el taller de formación profesoral.

\section{Pedro}

Cuadro 5: resúmenes de los textos reflexivos escritos por Pedro

\begin{tabular}{|c|c|}
\hline Inicial & Final \\
\hline $\begin{array}{l}\text { El tema abordado es el interés que siente por la } \\
\text { escritura en la universidad. Abarca dos etapas: una } \\
\text { anterior, como estudiante de Ingeniería; y una actual, } \\
\text { como profesor en la USB. Asimismo, detalla los } \\
\text { problemas que encuentra en su trabajo con los } \\
\text { estudiantes de pasantías: el excesivo uso de comas, la } \\
\text { utilización del lenguaje coloquial, la ausencia de } \\
\text { estructura en las ideas, etc.; finalmente, expresa su } \\
\text { deseo por recibir ayudas en el taller. }\end{array}$ & $\begin{array}{l}\text { Hace un balance en términos muy positivos de los } \\
\text { aprendizajes obtenidos en el taller de alfabetización } \\
\text { académica. Define de manera concreta las ayudas que } \\
\text { le han reportado las dos teorías abordadas. Para } \\
\text { finalizar, detalla cómo será su trabajo como tutor de } \\
\text { pasantías en la USB en términos de la planificación } \\
\text { que llevará a cabo para aplicar los conocimientos } \\
\text { adquiridos; y con ello, prevé en el futuro resultados } \\
\text { satisfactorios. }\end{array}$ \\
\hline
\end{tabular}

Sus argumentos en el texto inicial se basaron en un conjunto de anécdotas y en su aprecio personal por la escritura (en general). En cambio, en su escrito final, describe y explica exclusivamente aspectos de naturaleza epistemológica sobre la actividad de escribir en función de los conceptos clave vinculados a la escritura académica: el sistema de actividad y los géneros discursivos. Además, en el primer texto las referencias a la escritura se centraron en la ortografía, gramática, adecuación del léxico y coherencia. En contraste, en su último escrito estas referencias desaparecen. Asimismo, ya no emplea la metáfora de la escritura como recipiente o mero contenedor del mensaje que se desea transmitir. Esta referencia es remplazada por un concepto más amplio: la actividad de escritura. Por otra parte, sobre la enseñanza de la lengua se advierten cambios que acercan a Pedro al movimiento de la alfabetización académica. Por ejemplo, detalla cómo está poniendo en práctica los nuevos saberes para realizar con mayor pertinencia su trabajo de tutor de pasantías. Esto contrasta con su idea inicial de identificar y los corregir errores de escritura de 
sus estudiantes. Actualmente asume la situación como una tarea que le compete en su actividad profesional y que puede ser abordada mediante la metodología desarrollada en el taller.

\section{A modo de conclusión}

En cuanto a la ZDP colectiva, pensamos que la naturaleza de los datos analizados hasta el momento del envío de este artículo no permite avanzar conclusiones. Ahora bien, con base en el análisis interpretativo de los textos reflexivos escritos es posible verificar que la actividad educativa formal en alfabetización académica realizada en la USB tuvo para cada uno de los tres profesores un significado específico que derivó en cambios en el cumplimiento de su labor profesional. Así, las transformaciones fueron distintas en cada uno de ellos.

En síntesis, Pedro dio muestras de apropiarse de unos saberes que le permitieron modificar su pensamiento y actuación como profesor-tutor al incorporar nuevas herramientas de trabajo, específicamente, la teoría de la actividad y la de los géneros discursivos. Al terminar el taller, de los tres participantes, él es quien se ajusta más a los presupuestos de la alfabetización académica. Sobre Homero y Margarita, podemos señalar que aunque al finalizar la experiencia no manifestaron ni el conocimiento ni el uso práctico de las herramientas intelectuales ofrecidas en el taller sobre la alfabetización académica, sí consiguieron transformar en alguna medida su pensamiento: Homero logró la evaluación de su propio proceso como productor de textos académicos y valoró de un modo distinto a sus estudiantes en función de este proceso personal; de modo similar, Margarita cuestionó sus formas de actuación en el aula e incorporó cambios puntuales en relación con su propia escritura y la de sus estudiantes.

En definitiva, a partir de esta primera aproximación a los datos (que debe contrastarse y completarse con el análisis de los datos de las entrevistas colectivas realizadas durante las sesiones de trabajo) podemos decir que sólo en el caso de Pedro las transformaciones implicaron el aprendizaje expansivo propuesto como meta del taller, ya que los cambios reportados sobre la lengua escrita en el contexto académico le permitieron repensar y rediseñar de una manera más integral su actividad docente. Una explicación sobre estos resultados puede estar en la naturaleza misma de su actividad, en particular, en el rol que cumple en el sistema universitario de acuerdo con las reglas que lo regulan. Pedro se desempeña como tutor de informe de pasantía de final de carrera, de manera tal que el tipo de trabajo que desempeña propicia las transformaciones ocurridas en su pensamiento y 
actuación como docente que estuvieron mediadas por la reflexión y las experiencias del taller. En contraste, en los textos reflexivos de Homero y Margarita no es tan evidente su objetivo de acompañar a sus estudiantes en la tarea de convertirlos en escritores de un(os) determinados género(s) discursivo(s).

Es posible que gracias a esta condición de tipo contextual sigan observando la escritura como un conocimiento en general centrado en las reglas comunes a cualquier discurso, esto es, la ortografía, la gramática y el léxico, esencialmente. En este sentido, es posible pensar que una actividad docente universitaria que incluya entre sus objetivos la acción pedagógica de la alfabetización académica, como por ejemplo, mediante la renovación de los planes de estudio, nuevas políticas departamentales y la formación continua de los docentes, puede constituirse en un elemento de apoyo en el cumplimiento de los fines educativos en el contexto de la USB.

\section{Referencias bibliográficas}

Bajtín, M. (1989). Estética de la creación verbal. México: Siglo XX Editores.

Bazerman, C. (1994). Systems of genres and the enactment of social intentions. En A. Freedman, \& P. Medway, Genre and the new rhetoric (pp. 79-101). London: Taylor $\&$ Francis.

Borg, S. (2003). Teacher cognition in language teaching: A review of research on what language teachers think, know, believe, and do. Language Teaching, 36, 81-109.

Cambra, M. (2000). Introducció. El pensament del professor: formació per la pràctica reflexiva. En A. Camps, I. Ríos, y M. Cambra (coords.), Recerca i formació en didàctica de la llengua (pp. 161-171). Barcelona: Graó.

Engeström, Y. (1987). Learning by expanding: an activity-theoretical approach to developmental research. Helsinki: Orienta-Konsultit.

Engeström, Y. (1996). Los estudios evolutivos del trabajo como punto de referencia de la teoría de la actividad: el caso de la práctica médica de la asistencia básica. En S. Chaiklin y J. Lave (coords.) Estudiar las prácticas. Perspectivas sobre actividad y contexto (pp. 78-140). Cambridge: University Press.

Engeström, Y., \& Sannino, A. (2010). Studies of expansive learning: Foundations, findings and future challenges. Educational Research Review, 5, 1-24.

Latorre, A., del Rincón, D., \& Arnal, J. (1996). Bases metodológicas de la investigación educativa. Barcelona: Hurtado.

Palou, J. (2008). L'ensenyament i l'aprenentatge del català com a primera llengua a l'escola. Creences $i$ actuacions dels mestres amb relació a les activitats de llengua oral a l'etapa primària. Barcelona: Institut d'Estudis Catalans.

Palou, J. (2010). Metacognición y relatos de vida lingüística en los procesos de formación del profesorado. Análisis del relato de una alumna. En J. Herrera, M. Abril, y C. Perdomo (coords.), Diversidad cultural, plurilingüismo y estrategias de aprendizaje. Estudios sobre didácticas sobre las lenguas y sus literaturas (pp. 301-312). Tenerife: Servicio de Publicaciones de la Universidad de la Laguna. 
Palou, J. (2011). La construcción de textos reflexivos. Cuadernos de Pedagogía, 416, 63-67.

Palou, J., \& Fons, M. (2009). Actituds dels docents davant de les noves situacions escolars multiculturals i multilingües: qüestions recurrents. Zeitschrift für Katalanistik, 22, 151-169.

Pozo, J. I. (1999). Más allá del cambio conceptual: el aprendizaje de la ciencia como cambio representacional. Enseñanza de las ciencias, 17(13), 513-520.

Russell, D., Lea, M., Parker, J., Street, B., \& Donahue, T. (2009). Exploring notions of genre in academic literacies and writing across the curriculum: Approaches across countries and contexts. In C. Bazerman, A. Bonini \& D. Figueiredo (Eds.), Genre in a changing world. Perspectives on writing (pp. 395-423). Fort Collins/West Lafayette: Parlor Press.

Serrano, S. (2011). Escritura académica en la Universidad de los Andes: reflexiones sobre las prácticas en desarrollo. Legenda, 11, 78-97.

Serrón, S. (2004). Algunas reflexiones críticas acerca de la problemática de la enseñanza de la lengua materna en educación superior. Acción pedagógica, 13(1), 78-83.

Serrón, S. (2007). Presentación. En M. García Romero (coord.) Investigaciones sobre la escritura universitaria en Venezuela (pp. 2-17). San Cristóbal: Universidad de Los Andes-Núcleo Táchira.

Vigotsky, L.S. (1977). Pensamiento y lenguaje. Buenos Aires: La Pléyade.

\begin{abstract}
Referencia de la autora:
Isabel Martins es profesora e investigadora en el Departamento de Lengua y Literatura de la Universidad Simón Bolívar (Caracas-Venezuela) desde 2005. Anteriormente trabajó en la Universidad Central de Venezuela dictando cursos de formación lingüística en la Escuela de Derecho y en la Facultad de Ingeniería. En enero de 2014, obtuvo el título de Doctora en Didáctica de la Lengua y la Literatura, en la Facultad de Formación del Profesorado de la Universidad de Barcelona, gracias a una beca "Erasmus Mundus External Window Cooperation" de la Comisión Europea. También posee el Diploma de Estudios Avanzados en "Didáctica de la Lengua y la Literatura" de la Universidad Autónoma de Barcelona. Sus trabajos de investigación se han centrado en el pensamiento del profesor y la escritura académica. Desde 2008, gestiona el blog http://leeryescribirenlausb.blogspot.com.es para brindar apoyo didáctico a los estudiantes uesebistas en los cursos de "Lenguaje" impartidos durante el primer año de carrera.
\end{abstract}

Email: imartins@usb.ve

i Este texto deriva de la investigación titulada: "La formación profesoral en alfabetización académica en la Universidad Simón Bolívar: un estudio de caso de las transformaciones en las creencias, representaciones y saberes de unos profesores sobre la escritura académica". Se trata de la tesis doctoral en Didáctica de la Lengua y la Literatura que, para el momento de envío de este escrito, realizo en la Universidad de Barcelona (España). Su director es el Dr. Juli Palou Sangrà, coordinador del grupo de investigación PLURAL, Plurilingüismos Escolares y Aprendizaje de Lenguas, y profesor titular de la Facultad de Formación del Profesorado de esta casa de estudios.

Para citar este artículo:

Martins, I. (2014). Transformaciones en el pensamiento sobre la escritura académica de unos profesores universitarios de áreas no lingüísticas. Bellaterra Journal of Teaching \& Learning Language \& Literature, 7(2), 21-33. 